\title{
5 Der lange Weg zur persönlichen eAkte: Digitalisieren, strukturieren, qualifizieren und dann elektronifizieren
}

\author{
Stefan Müller-Mielitz
}

\section{Bestandsaufnahme und Stellenwert}

Die Dokumentation in deutschen Krankenhäusern ist nach wie vor papierbasiert. In den letzten Jahren wurden elektronische Patientenakten aufgebaut, die elektronische Daten beinhalten. Diese eAkten sind aber institutionelle Akten, deren DatenbankSystem proprietär und damit für einen Datenaustausch zwischen Leistungserbringern (intersektoral oder interregional) nicht geeignet ist.

Durch das Scannen von Papierbelegen (= digitalisieren) werden die aus der Papierakte erzeugten Digitalisate analog zu originär erzeugten elektronischen Formularen und Dokumenten in einem Dokumenten-Management-System (DMS) anzeigbar. Auch hier ist ein Datenaustausch von einzelnen medizinischen Werten nicht möglich. Die Systeme sind institutionell gebunden und proprietär.

Das deutsche Gesundheitswesen arbeitet zum jetzigen Zeitpunkt in der stationären Versorgung mit hybriden Systemen, da es Krankenhäuser gibt, die elektronische Dokumente vorhalten und nebenher mit einer reinen Papierakte arbeiten oder diese Papierakte nach der Behandlung digitalisieren und damit arbeitsplatzunabhängig im DMS zur Verfügung stellen. Die arbeitsplatzunabhängige Präsentation der dDokumente ist der Hauptnutzen der Digitalisierung durch Scannen. Marktbeobachtungen gehen davon aus, dass ca. 300 Krankenhäuser noch gar nicht scannen. 
5 Der lange Weg zur persönlichen eAkte: Digitalisieren, strukturieren, qualifizieren und dann elektronifizieren

\section{Beschreibung des Transformationsprozesses}

Ziel des Digitalisierungsprozesses muss es sein, elektronisch erzeugte Daten, technisch standardisiert und semantisch interoperabel austauschen zu können, was als Elektronifizierung bezeichnet werden kann. Von der eSource bis zur eArchivierung ist der Dokumentenlifecycle künftig komplett elektronisch. Ein möglicher Weg ist der Dokumentenaustausch mittels Clincal Document Architecture (CDA) und auf technischer Kommunikationsebene mit IHE-Profilen (XDS und XDS-SD). Aktuelle Projekte schaffen dieses bereits, indem sie digitalisierte Dokumente und elektronische Dokumente auf den standardisierten IHE-Weg bringen. Auch hier ist ein Datenaustausch von medizinischen Daten noch nicht möglich, da nur Dokumente standardisiert übertragen werden. Dieses wird erst in der künftigen Phase der detaillierten Elektronifizierung möglich sein in Kombination von medizinischen Daten mit semantischer Codierung. Dazu müssen die jetzigen papierbezogenen Dokumente und bereits vorhandene elektronische Dokumente im Detail seziert werden und medizinisch-fachlich kodiert werden. Dann wäre ein detaillierter Datenaustausch möglich.

\section{Status Quo}

Digitalisate werden in Dokumenten-Management-Systemen angezeigt und werden damit auf die Ebene eines elektronisch erzeugten Dokuments gehoben. Angelehnt an die Struktur und Hierarchie der Papier-Patientenakten finden sich alle Nutzer in dieser hierarchisch organisierten digitalen Akte schnell zurecht. Die digitale Akte ist damit eine zusammengeführte Akte, die doppelte Dokumente beinhalten kann und deren Vollzähligkeit (sind bestimmte Dokumente in der Akte vorhanden?) nicht geprüft ist. Erfolgt dieser Qualifizierungsschritt steht dem Nutzer eine konsolidiere Akte zur Verfügung. Ein Datenaustausch von medizinischen Detaildaten ist dann aber noch nicht möglich.

\section{Chancen und Risiken}

Eine konsolidierte digitale Akte, bestehend aus digitalisierten und originär elektronisch erzeugten Dokumenten bietet für die Institutionen im Gesundheitswesen die Möglichkeit, die bestehenden Papier-Workflows auf elektronische Workflows zu ändern. Die beteiligten Nutzer und Nutzerinnen werden sich an diese elektronischen Prozessänderungen langsam gewöhnen, sie lernen den Umgang mit elektronischen Medien kennen. Damit beginnt der Transformationsprozess auf organisatorischer Ebene.

Da die eProzesse neu und ungewohnt sind, die Daten nun elektronisch vorliegen und damit anders als eine Papierakte überall verfügbar sind, bestehen auch Gefahren im Umgang mit diesen sensiblen Dokumenten und den beinhalteten Daten. Es wird dazu viel Aufwand in die Sicherheitsinfrastruktur der Institutionen gelegt werden müssen, es sind Softwareanpassungen von den IT-Herstellern durchzuführen, die Sicherheit und Nutzungskomfort vereinen. Den Institutionen im Gesundheitswesen und den Nutzern und Nutzerinnen wird dadurch das Thema Compliance noch stärker in den Vordergrund gestellt neben Datensicherheit, Datenschutz und Risikoabwägungen. 


\section{Trends und Entwicklung}

Neben den organisatorischen Anpassungen in den Institutionen des Gesundheitswesens sind auch detaillierte technische Anpassungen notwendig. Zurzeit gibt es noch keine konsolidierte digitale Akte für einen elektronischen Datenaustausch von medizinisch relevanten Daten. Selbst der elektronische Austausch von Dokumenten ist nicht in der Breite möglich. Von der Seite aus betrachtet ist der Digitalisierungsgrad gering, wenn nur wenige Dokumente elektronisch ausgetauscht werden und nach wie vor das Fax und der Postbrief die Wahl der Kommunikation sind. State-ofthe-Art-Verfahren zur Bestimmung des Digitalisierungsgrades berücksichtigen die folgenden Punkte noch nicht:

Der Transformationsprozess von Daten in standardisierte, interoperabel Dokumente, die dann in intersektoralen und interregionalen und nationalen und künftig auch internationalen Gesundheitstelematik-Infrastrukturen von einem IT-System in ein anderes nicht nur übertragen sondern auch auf Basis von internationalen Codes und standardisierter Semantik auch wieder einlesbar und weiterverarbeitbar sind.

Diese Schritte müssen in naher Zukunft angegangen werden, damit das Ziel einer einrichtungsübergreifenden elektronischen Patientenakte, die dem Wohl des Patienten dient und die unter seiner persönlichen Regie steht, erreicht wird.

\section{Vom Papier bis zur persönlichen einrichtungsübergreifenden Patientenakte} (s. Abb. 1)

Die Dokumentation in modernen Krankenhäusern befindet sich technisch auf der Stufe der digitalen Akte. Diese wird befüllt durch digitalisierte Dokumente und eDokumente. Die Akte ist damit für dDokumente nur auf Klassen und Registerebene durchsuchbar. Sie enthält auch Dubletten. Die Akten und Dokumente werden durch das Dokumenten-Management-System präsentiert. Die in den Krankenhaus-Informations-Systemen gehaltenen Daten sind proprietär und als institutionelle elektronische Akten (iePA) zu bezeichnen. Parallel dazu sind die in den DMS angezeigten Dokumente teilweise auch durch OCR-Container durchsuchbar. Einzelne IHE-basierte Projekte erarbeiten Akten mit den IHE-Profilen XDS und XDS-SD (SD steht für Scanned Documents). Um als konsolidierte Akten

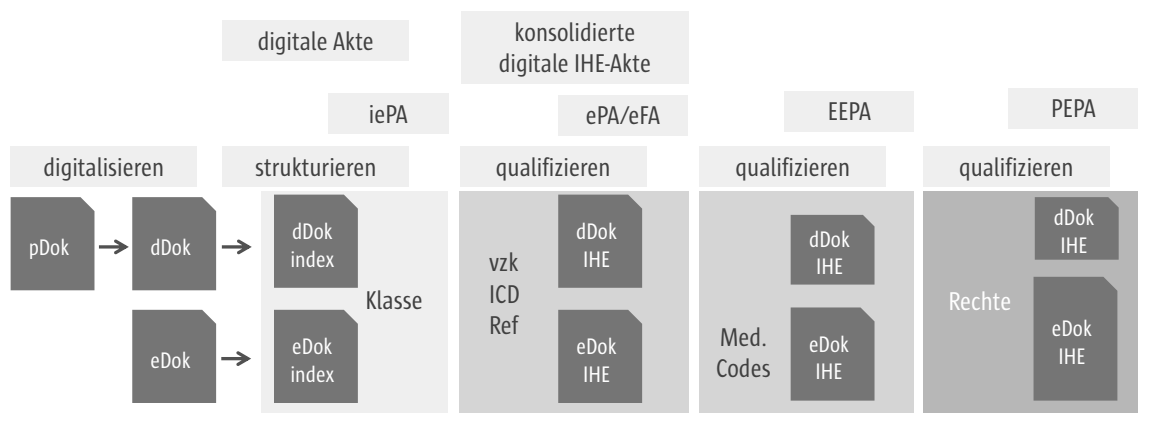

Abb. 1 Vom Papier (links) bis zur persönlichen einrichtungsübergreifenden Patientenakte 
5 Der lange Weg zur persönlichen eAkte: Digitalisieren, strukturieren, qualifizieren und dann elektronifizieren

zu gelten, müssen diese IHE-Akten eine Dublettenprüfung und eine Vollzähligkeitsprüfung durchlaufen. Die einfache Vollzähligkeit (bestimmte Dokumente müssen in der Akte sein) und die komplexe Vollzähligkeit (ICD- und OPS-Code erfordern das Vorhandensein bestimmter Dokumente). Dieses erfolgt beispielsweise im FALKO.NRW-Projekt. Die so qualifizierten Akten können intersektoral und interregional wie im FALKO-Projekt über die elektronische Fallakte ausgetauscht werden. Auch diesen Akten fehlt dann die Möglichkeit, einzelne Daten in unterschiedlichen IT-Systemen zu nutzen. Dazu ist die Qualifizierung bezüglich der medizinischen Codes (wie LOINC, SNOMED und weiterer) notwendig. Erst dann kann von einer einrichtungsübergreifenden intersektoral nutzbaren elektronischen Patientenakte gesprochen werden. Dieser Schritt der Elektronifizierung von Dokumenten mit einer medizinisch-fachlichen Kodierung ist der dann vorläufig letzte Schritt beim Aufbau von E-Akten. Diesen Akten fehlt dann, da sie aus der Institution betrieben werden und arztgeführt sind, nur noch die Möglichkeit aus Patientensicht entsprechende Rechte zu vergeben, sodass dann in einem letzten Schritt die selbstbestimmte persönliche, einrichtungsübergreifende Patientenakte (PePA) entsteht. Der lange Weg hin zur Elektronifizierung des Gesundheitswesens ist dann vollzogen. 